\title{
Gut microbiome diverges quickly with rapid host evolution in Trinidadian guppies
}

\author{
Sarah Evans ${ }^{1}$, Eugenia Zandona ${ }^{2}$, Jeferson Amaral $^{2}$, and Sarah Fitzpatrick ${ }^{1}$ \\ ${ }^{1}$ Michigan State University \\ ${ }^{2}$ Universidade do Estado do Rio de Janeiro
}

November 25, 2020

\begin{abstract}
We took advantage of a five-decade 'chronosequence' of host-microbiome evolution to examine changes in the gut microbiome as wild populations evolve. In an iconic example of rapid evolution in the wild, Trinidadian guppies have displayed parallel phenotypic convergence when translocated six times from high predation (HP) to low predation (LP) environments, but changes in microbiome are unknown. We find microbiomes of fish translocated 5-6 years ago were already more similar to LP native populations than HP sources, and attribute this to both environmental and host-morphological changes. While diet was a minor driver of microbiome structure, we suggest a nutritional role for the gut microbiome in guppy evolution whereby nitrogen-fixing bacteria supplement the low-nutrient diet in LP environments. We show that environment and rapid phenotypic shifts in gut traits interact to control microbiome assembly, and suggest the gut microbiome plays an important but not necessarily consistent role in rapid host evolution.
\end{abstract}

\section{Introduction}

The diverse community of symbiotic microorganisms comprising an organism's microbiome is recognized as a crucial component of its physiology (Rawls et al. 2006; Li et al. 2008; Bordenstein \& Theis 2015) and behavior (Vuong et al. 2017). The microbial community that occupies an organism's gastrointestinal tract (i.e., the gut microbiome) has been shown to be an especially important determinant of host fitness as it can regulate resource acquisition (Zhu et al. 2011), life history (Gould et al. 2018), and disease resistance (Lathrop et al. 2011). As such, the diversity and composition of the gut microbiome is emerging as an important aspect of the 'holobiont', or host-microbiome system (sensuZilber-Rosenberg \& Rosenberg (2008)), that can itself be under strong natural selection. Determining the function of the gut microbiome (i.e., its effect on host physiology, health, and ultimately, evolutionary fitness) and the factors that shape diversity and composition of this community are two highly sought-after goals (Benson et al. 2010; Spor et al. 2011). While there are some accounts of conserved, core, microbiomes for particular species (Roeselers et al. 2011), determining the primary controls on microbiome composition is difficult because in nature gut microbiomes vary widely across individuals (Turnbaugh \& Gordon 2009), space (Smith et al. 2015), and time (Nayak 2010). We use an unprecedented five-decade experiment to study the environmental and host-driven controls on gut microbiome and their potential to shape adaptive differentiation in the wild.

Bacteria enter the gut vertically from mother to offspring (Milaniet al. 2015; Beemelmanns et al. 2019) and horizontally through contact with the environment and food (Nayak 2010). Introduced taxa may be transient if they pass through the gut or die, or they can establish and become part of a self-perpetuating population. Thus, a gut community is determined by the availability of microbial colonizers in an environment, and 
filtering of those colonizers by host traits or genetics, and conceivably, could assemble in parallel with similar selection pressures and similar environments.

Host diet influences the gut microbiome through both colonization, as food carries microorganisms into the gut, as well as filtering, since diet can drive evolution of digestive traits. Studies at large taxonomic scales suggest diet is a stronger control on microbiome structure in the latter scenario, when it affects traits. For instance, the gut microbiomes of pandas are more similar to closely-related carnivores than distantlyrelated herbivores, presumably because their gut morphology evolved from a carnivorous ancestor (Karasov \& Douglas 2013). The gut microbiome may be an especially strong modulator of host fitness in circumstances when there is a mismatch between traits and function - that is, host physiology does not allow for digestion of certain resources abundant in a new environment (e.g. cellulose in termites and plant toxins in mammalian herbivores), but the gut microbiome does (Abe et al. 2000; Kohl et al. 2014).

This mismatch among environment, host traits, diet, and microbiome is likely a consistent feature of hostmicrobiome co-evolution in the wild, but impossible to capture in most systems, which do not control for genetic background or examine populations that have already diverged. Studies can control for genetic background by rearing populations in controlled environment, but this excludes the complex way that drivers interact in nature (e.g. behavior and habitat interact to determine diet). Our study achieves an optimal middle-ground to understand the role of gut microbiomes in local adaptation: a 'natural laboratory' where replicate wild populations of known origin have undergone parallel phenotypic evolution to novel environments (Reznick et al. 1990, 1997; Gordon et al. 2015). In this system, populations of guppies (Poecilia reticulata) originating from high predation (HP) Trinidadian streams were introduced to guppy-free low predation (LP) sites six times between 1957 and 2009 (Haskins, unpublished data, Endler 1980; Reznick \& Bryga 1987; Travis et al. 2014). We capitalized on this time series of guppy transplant experiments conducted over the last five decades to understand the determinants of guppy gut microbiome structure and function.

Microbiome structure is likely to be shaped by several factors as high predation (HP) guppies evolve to low predation (LP) environments, several of which have been documented in native HP and LP ecotypes. First, LP environments are at higher elevations and tend to have less sunlight due to higher canopy cover and lower primary productivity (Zandonà et al. 2017). Thus, transplanted guppies may be exposed to a different pool of microorganisms to colonize their gut microbiome (Nayak 2010). As these environmental differences alter the types of resources available, guppies in LP environments eventually adopt a more omnivorous, lower-quality diet than HP guppies, which eat more nutrient-rich macroinvertebrates abundant in these sites (Zandonà et al. 2011). Finally, these differences in diet likely underlie observed differences in guppy gut length: LP populations have longer guts than HP populations (Zandonà et al. 2015), presumably to maximize nutrient absorption and energy extraction from lower-quality food types in lower-resource LP environments (Kapoor et al.1976).

Taking advantage of this 'chronosequence' of host evolution, we hypothesize that gut microbiota tracks the evolution of fish traits such as gut morphology, such that both will change linearly with time as populations inhabit a new predation regime. Previous work shows that gut microbiomes diverge across ecotypes, but not necessarily in parallel, perhaps due to stream-specific effects (Sullam et al. 2015). However, in this same study, gut microbiomes from HP and LP fish remained distinct even after being fed 10 weeks of the same diet in the lab, suggesting that host traits, genetic background, or high vertical transmission also controls the microbiome. If our hypothesis is supported, guppy gut microbiome of recent introductions (introduced to new LP environments 5-6 years prior to sampling) will be compositionally more similar to HP ecotypes, while gut communities sample from older introductions (30-60 years prior to sampling) will be more similar to LP native microbiomes because they have had more time to evolve predictable LP traits such as longer gut length. 


\section{Methods}

\section{Sample collection and site characterization}

In the Northern Range Mountains in Trinidad, guppies originating from high predation (HP) environments have been previously transplanted into guppy-free, low-predation (LP) environments, and monitored as they evolved towards the LP guppy ecotype, forming one of the best examples of replicated parallel evolution in the wild to date (Reznick et al. 1996; Magurran 2005; Gee et al. 2009). Translocations occurred in six instances $(1957,1976,1981,2008$, and for two streams in 2009) across five drainages (Aripo, Campo, El Cedro, Guanapo, Turure; Fig. 1). In February 2014, we field-collected guppies from each of these six transplant sites, which we call "Introductions" (LP environments where HP populations were previously introduced). We also collected fish from the three original HP populations that served as sources for the introductions, and from three native LP populations as presumed locally adapted references (Fig. 1). We divided sites into four categories: HP source, LP native, old introduction (introduced 30-60 years prior to sampling), and recent introduction (5-6 years prior to sampling).

We collected 19-35 female guppies from each of these 12 sites. We sampled three distinct pools that were at least $30 \mathrm{~m}$ apart to capture within-site heterogeneity, and only females to control for possible sex differences (Bolnick et al. 2014b) and for comparability to Sullam et al. (2015). Sampling was lower at Naranjo (N=19) because rain limited sampling time and Tumbasson $(\mathrm{N}=21)$ because of its small population size. We weighed and euthanized individuals with an overdose of MS-222 immediately after field capture, and preserved them in $95 \%$ ethanol. We measured stream $\mathrm{pH}$ and temperature using a handheld meter (Hanna Instruments, Smithfield, RI, USA), and recorded GPS coordinates of each pool. Fish condition was calculated as weight divided by length.

\section{Characterization of gut length and content}

In January 2015, 250 guppies preserved in ethanol at $-20^{\circ} \mathrm{C}$ were dissected with sterile instruments in the laboratory. The tubular digestive tract was removed, washed in $70 \%$ ethanol, measured for gut length with a ruler in a sterile petri dish, and cut to delineate anterior and posterior sections. Anterior and posterior guts were preserved in ethanol and DNA was extracted within 2 weeks. A separate set of 105 intact guppies were shipped to Universidade do Estado do Rio de Janeiro for analysis of gut length and gut content. Upon dissection, guts were removed and preserved in $10 \%$ formalin, then weighed, photographed and measured using the software ImageJ (Schneider et al. 2012).

Methodology used for gut content analysis was the same used in Zandonàet al. (2011). Briefly, we analyzed the first third of each gut, which included the stomach and a small part of the foregut, because here items are not fully digested and thus identifiable. The gut contents were then distributed evenly onto a 64 -square gridded slide squares and examined under a dissecting scope to identify invertebrates to the lowest possible taxonomic degree, generally the family (Mugnai et al. 2010). The slide was then analyzed under a compound microscope, where 10 squares were randomly chosen among the 64 to estimate the area covered by each food item: invertebrates, detritus, diatoms and filamentous algae. Detritus and filamentous algae areas were estimated for each square, while diatoms were counted. We assigned an average size for diatoms to calculate the total area occupied in each square. Due to the patchier distribution of invertebrates on the gridded slide and their lower occurrence but bigger sizes, we estimated the area covered by them in all 64 squares. Subsequently, we estimated the total area and relative percentages covered by each food item in the whole slide. Invertebrates are the most nutritious item and, together with detritus, they made up most of the gut contents. Because invertebrate and detritus percentages were correlated, we only considered invertebrates in the statistical analysis.

\section{Characterization of gut microbiome}

The adhesion and colonization of the microbiota inside fish guts may be influenced by several factors linked to the stomach, pyloric caeca, and intestine portions (Ring $\varnothing$ et al., 2003). Thus, we evaluated the microbiome in both the anterior and posterior portion of the guppy intestinal tracts. We extracted DNA from anterior 
and posterior sections ( $250 \times 2=500$ total) following the manufacturer's protocol of the MoBio PowerSoil DNA Extraction kit. We submitted DNA to the Michigan State University Core Genomics Facility for Illumina sequence library construction using the Illumina TruSeq Nano DNA Library Preparation Kit and sequencing. We submitted a total of 508 samples for sequencing, randomized across two lanes. Eight samples (four anterior and four posterior) were included in both lanes to serve as controls. Following the core facility's standard protocols, bacterial 16S V4 (515f/806r) Illumina compatible libraries were prepared using primers containing both the target sequences and the dual indexed Illumina compatible adapters (see Kozich et al. 2013). Completed libraries were normalized using Invitrogen SequalPrep DNA Normalization plates, pooled and cleaned up using AmpureXP magnetic beads. One sample failed to amplify so 507 samples were sequenced. $16 \mathrm{~S}$ amplicon pools were sequenced independently in a $2 \times 250 \mathrm{bp}$ paired end format using independent v2 500 cycle Illumina MiSeq reagent cartridges (Illumina, CA, USA).

Bacterial and fungal bioinformatics pipelines are fully described in Appendix S1. Briefly, reads were quality filtered and merged using the USEARCH pipeline (http://drive5.com/usearch/). Primers and adapter bases were removed using cutadapt (Martin 2011). Bacterial reads were filtered and truncated to $250 \mathrm{bp}$, clustered into operational taxonomic units (OTUs) at 97\% identity level then classified against SILVAv123 rRNA database (Yilmaz et al. 2014). Sequences were aligned and a phylogenetic tree was built using PASTA (Mirarab et al. 2015). We removed (i) OTUs classified to Chloroplast and Mitochondria, ( $i$ ) OTUs present in less than 5 (of 507) samples, (iii) OTUs that had less than 20 reads across all samples, and (iv ) samples with less than 2000 reads (Table S1). For calculating diversity metrics, we rarefied samples to 8000 reads. Bioinformatic code is publicly available (https://gitlab.msu.edu/belldere/guppy_gut_2015).

\section{Data analysis}

Our general modeling framework included site category as a fixed effect (HP source, LP native, old introduction, and recent introduction) and site as a random effect nested within drainage (Fig. 1). We assigned drainages to reflect paired HP to LP translocations. For example, although the El Cedro is a tributary of the Guanapo River, we assigned the two El Cedro sites their own drainage because the translocation occurred within that river. Turure was assigned to its own drainage (as opposed to its HP source site Guanapo) because these two sites are not physically connected. We used this model structure to test for differences among all univariate response variables (stream characteristics, fish traits, gut content) with linear mixed-effects models (R package lmer, Bates et al. 2015), after transforming response variables to meet assumptions of normality, comparing null and fixed-effect models using a likelihood ratio test.

Before testing statistical differences among gut microbial communities, we performed standard pre-processing steps (Table S1). MiSeq lane did not significantly affect the composition of our control samples (PerMANOVA $\mathrm{p}<0.1)$. We assessed statistical differences in community structure across site categories using permutational analysis of variance (PerMANOVA) on the unweighted (presence-absence) and weighted (incorporates abundance) Unifrac distance matrices using phyloseq (McMurdie \& Holmes 2013) and vegan (Oksanen et al.2017) in R (R Core Team 2017). Permutations were robust to the order of factors, which included gut position, stream treatment, and site, and were constrained by drainage as a random effect. We also tested the effect of site category on posterior gut microbiomes (all sites), in LP sites alone (LP natives and introductions), and within each drainage, adjusting p-values using Bonferroni correction.

We quantified the variance in community composition explained by environmental variables (stream temperature, which correlated to stream $\mathrm{pH}, \mathrm{r}^{2}=0.42, \mathrm{p}<0.001$ ), gut morphology, and gut content using distancebased redundancy analysis (function dbRDA in vegan, Legendre and Anderson 1999). DbRDA regresses predictor variables on a sample dissimilarity matrix and tests their significance for explaining community distances. Our model included drainage as a conditional factor. We partitioned the variance each predictor variable explained using multivariate variance partitioning (function varpart, vegan). Compared to the commonly-used envfit function, dbRDA and varpart partition variance from the raw distance matrix, rather than correlating variables with reduced data, which predisposes predictors to explain a high degree of variance. Gut content analysis (which also included gut length measurements) was performed on a subset of fish distinct from the subset for which gut microbiome was sequenced. Therefore, diet was described at the 
population level rather than paired to microbiome measurements. To account for this, we used populationlevel means for all predictor variables. This did not affect conclusions. Since the microbiome from HP source populations was so distinct from other site categories, we repeated all variance partitioning and dbRDA without HP source to examine factors controlling mirobiome in LP environments.

We used the distance matrices to calculate mean distance between a source population and its respective introductions (and the LP native site from the corresponding drainage). Betadispersion, or the compositional variation of the microbiome among a group of individuals, was calculated using betadisper in vegan (R Core Team 2017). In addition to betadispersion, we quantified microbiome richness, Shannon-Weiner diversity (Pielou 1975), and Pielou's Evenness (Pielou 1975) on rarefied data. We assessed differences using the univariate linear mixed effects models described above.

\section{Results}

Environmental differences among HP and LP environments varied mostly as expected based on what is already known about Trinidadian streams. Namely, lower elevation, high-predation (HP) sites had higher stream water temperature (HP: $24.87^{\circ} \mathrm{C}, \pm 0.08$ vs. LP: $\left.22.82^{\circ} \mathrm{C}, \pm 0.03\right)$ and $\mathrm{pH}(\mathrm{HP}: 7.5 \pm 0.03$, LP: 6.7 $\pm 0.03)$ compared to low-predation sites $(\mathrm{p}<0.01$ for both comparisons), which include LP native, old, and new introductions (see Fig. S1 for site-level stream data). Fish morphology also fit expectations: fish in LP environments had longer gut lengths relative to body size (Fig. 2A) and were larger overall (Fig. 2B). Site-specific variation overwhelmed significant effects of site category on gut content (as measured by percent invertebrates, Fig. 2C), and fish condition (weight/length, Fig. 2D). If anything, at the time of sampling, LP fish were eating more invertebrates (Fig. S1D), contrary to expectations. HP fish had diets richer in detritus, as well as higher relative abundance of cyanobacteria (according to $16 \mathrm{~S})$ in their guts $(\mathrm{p}<0.001$, Fig. S2, Table S4).

After pre-filtering steps (Table S1), we identified 12,068 bacterial OTUs in 485 guppy gut microbiome samples. In general, the posterior portion of the gut harbored bacterial communities that were more diverse (Fig. S3) and compositionally distinct from the anterior portion (PerMANOVA $\mathrm{p}<0.001$ ). Across site categories, however, gut position explained less variation than other factors (Table 1), and differences in diversity and composition across site categories were similar in anterior and posterior communities (e.g. Fig. S2, S4, S5). Thus, we focused remaining analyses on the posterior gut microbiome, which may be less transient than the anterior gut community.

Site category had a significant effect on gut microbiome community composition (Fig. 3A; PerMANOVA $\mathrm{p}=0.001$ ). The presence or absence of bacterial taxa in the gut was largely driven by stream water temperature (which correlated closely to stream $\mathrm{pH}$ ) and gut length (Fig. 3 inset, Table S2). When HP source populations were removed and only LP environments were considered (LP native, old, and recent introduction sites), diet was a larger contributor to microbiome composition (Fig. 3B), but not in the dimension that corresponded with site category (arrows in Fig. 3B), for which water temperature was more important. Diet also had elevated importance when changes in relative abundances of taxa were accounted for (Fig. S6), compared to presence-absence alone, as also found by Sullam et al. (2015).

There were larger differences between gut microbiomes of HP source populations and those in LP environments (i.e. LP native and introduction sites) than among LP sites. When we compared microbiomes in LP environments to microbiomes of their respective HP source populations, microbiomes of introductions (e.g. Intro 2008, 2009, 1957, etc.) more closely resembled each other, and LP native populations, than their respective HP source site (Fig. 4, distance from 0 in y-axis). Gut microbiomes in introduced populations sometimes, but not always became more similar microbiomes of reference LP native populations (Fig. 4 does not convey this, but see Fig. S7). In two of four drainages, gut microbiome composition of introduced populations remained significantly different from those of LP native sites (Fig. S7, Table S3), with no effect of time since introduction. 
Variation in microbiome composition across individuals was highest in HP sites (Fig. S8, betadispersion test $\mathrm{p}<0.01$ ), and lower in the introductions than native sites, even though we might expect it to be higher in more genetically varied populations. Higher betadispersion was not driven by a greater among-individual diet diversity ( $p>0.05$ for correlation, data not shown). While betadispersion differences can influence betadiversity tests (i.e. PerMANOVA), these differences were small so not likely to be the sole reason we observed significant differences in composition (Anderson \& Walsh 2013).

Mean number of species within individual guts also differed across site categories. Gut bacterial richness was significantly lower in native populations (HP source and LP native) and higher in introductions (Fig. 5A), in both anterior and posterior gut positions (Fig. S4), while community evenness and alpha diversity were less responsive (Fig. S4). Richness was positively correlated to relative gut length $\left(\mathrm{r}^{2}=0.43, \mathrm{p}<0.001\right)$, which may explain previously-observed positive correlations between fish length and microbiome diversity (Bolnick et al. 2014a; Forberg et al.2016), and was not explained by higher diet diversity (Bolnick et al. 2014a).

Gut communities were dominated by Proteobacteria, Actinomycetes, and Firmicutes (Fig. S2), similar to other fish microbiomes (Sullam et al. 2012), and previous dominant phyla in guppies at this site (Sullamet al. 2015). Changes in the relative abundances of the most dominant taxa (Table S4) largely drove trends in weighted Unifrac betadiversity. Five of the 10 most abundant taxa across the entire dataset were from the order Rhizobiales, a genus able to fix nitrogen to create nutrients. While we did not measure fitness, we did observe patterns that suggest this additional source of nutrients could have increased fitness. For instance, the significantly more abundant Rhizobiales species in El Cedro (Fig. S9) could explain why these fish were in better condition than their LP native counterparts, despite a lower quality diet (Fig. S1D,E). The total Rhizobiales abundance (Fig. 5B, S9), as well as the abundances of these highly abundant taxa (Fig. 5C), tended to be highest in the old introduction streams, and lowest in HP source streams.

\section{Discussion}

\section{Consistent divergence and drainage-specific convergence of gut microbiomes}

We investigated variation in the guppy gut microbiome along an evolutionary 'chronosequence' of time-sinceintroduction, hypothesizing that gut microbiota tracks the evolution of fish traits such as gut morphology. Our hypothesis was supported in that, when HP fish were introduced into LP sites, their gut morphology changed to resemble that of LP native populations, and their microbiomes tracked this change. Coevolution of host and microbiome has been observed in macroevolutionary studies (Ley et al. 2008; Muegge et al.2011), but not always in replicate, independent divergences in the wild (Sevellec et al. 2018; Härer et al. 2020). We uniquely show that coevolution not only occurs but can be quite rapid; in our study, HP guppies translocated to LP environments after 5-6 years (approximately 15-18 guppy generations) were already more similar to native LP fish, despite their genetic background being more similar to their HP source populations (Fitzpatrick et al. 2015). This rapid microbiome divergence was driven by shifts in phenotypic traits and environment, suggesting that these factors are equally important for driving microbiome as diet and host genetics, which are more commonly studied.

While we observed dramatic and consistent divergence from HP microbiomes toward LP native microbiomes, even in the first 5 years after translocation, we did not observe consistent convergence of introduced populations with LP natives in every drainage. Although we cannot be sure, lack of convergence with LP natives does not appear to due to insufficient time. Some of the most recent introduced populations were indistinguishable from LP native microbiomes (Guanapo drainage), while one of the oldest introductions (El Cedro, 57 years in LP environment) remained distinct from LP natives. Abiotic differences among sites most strongly explained why LP and introduced microbiomes were not more similar. But while these site-specific effects led Sullamet al. (2015) to rule out parallel microbiome co-evolution in this system (because LP populations did not converge) our larger study suggests this should not be ruled out. Rather, the consistent divergence from HP source, drainage-dependent convergence with LP natives, and potential mechanisms for 
microbiomes to impact nutrition (see third section) suggest the gut microbiome affects host evolution while in the context of other agents influencing microbiome assembly (e.g. horizontal transmission). We further point out that parallelism at the functional level could occur without documenting consistent parallelism at the taxonomic level, especially given high functional redundancy in microbial communities (Louca et al. 2017).

\section{Stream characteristics and gut morphology drive microbiome composition}

Abiotic stream characteristics were strong drivers of gut microbiome. Since temperature and $\mathrm{pH}$ are known drivers of microbial community composition in freshwater (Fierer et al. 2007), such a correlation might suggest that these factors are influencing gut microbiome by exerting selection on the species pool (stream microbiota) that colonizes the gut. However, this is not supported by Sullamet al. (2015), who found little overlap between guppy gut microbiomes and their respective stream water. Alternatively, abiotic variables could shape gut microbiome through other processes than selection on the stream species, such as influencing prey, and their associated microbial colonists (Jacobsen et al. 1997; Smithet al. 2015). While the mechanism of how exactly stream properties affect microbiome is unresolved, it does suggest that vertical transmission from parent to offspring does not overcome the effect of site-specific factors on gut assembly, even in livebearing species.

An equally important driver of microbiome composition was gut length, which, like the microbiome, shifted from the typical HP phenotype to more closely resemble the LP phenotype, even in recent introductions. Gut length is known to diverge in parallel between HP and LP ecotypes (Zandonà et al. 2015) as more omnivorous LP guppies evolve longer intestines to help with nutrient absorption in the lower-resource LP environment. While adaptive evolution has been shown to occur rapidly in this system (Reznick et al. 2019), the change in gut length may also reflect phenotypic plasticity. Zandonà et al. (2015) previously showed that differences between HP and LP guppy gut lengths are smaller during the wet season when HP are more omnivorous. This trait may be phenotypically plastic in other organisms, particularly under fluctuating resource availability (Grether et al. 2001; Keet al. 2008), since digestive tissues are costly to maintain and build (Sibly et al. 1981). Regardless of the mechanism by which guts grew longer in LP environments, our study suggests that phenotypic change in host traits drives microbiome change. Since both stream properties and gut length had already shifted in the most recent introductions (5-6 years in novel environment), we could not parse out which was a stronger driver of gut composition. Future studies could ask how the microbiome impacts host colonization of new site by capturing generations immediately after translocation (or invasion), and also by characterizing environmental microbiome, like those in water and on prey.

Diet can alter gut microbiome in two ways: through changes in the materials entering the gut, which affect microbiome colonization and filtering, and through nutritional changes, which alter digestive traits like gut morphology and chemistry. In our study, gut content played a small role in driving microbiome composition, compared to shifts in gut morphology. This is supported by previous work on guppies, which showed that HP and LP fish retain different microbiomes even when fed the same diet (Sullam et al. 2015), and at broad taxonomic scales, where microbiome divergence track diet primarily in accordance with evolution of in digestive traits (e.g. hindgut to foregut) (Muegge et al.2011). Gut content may primarily affect microbiome (particularly the relative abundance of taxa) when gut morphology is held constant, or when gut content matches aggregated diet. Gut content may not have reflected typical HP and LP diets in our study due to time of sampling (Zandonà et al. 2015, 2017). At the same time, if seasonal diet shifts result in plastic morphological changes, this could be equally powerful in altering gut microbiome. As our understanding of microbiome divergence begins to extend beyond controlled studies, it will be important to capture the complexity of diet in the wild, which may be temporally dynamic (Colston \& Jackson 2016), influenced by complex behaviors, and not easily characterized by single axes induced in the laboratory.

\section{Microbiome may facilitate host evolution by overcoming nutritional barriers}

The changes in microbiome that we identified may affect microbiome function, fish metabolism, and ultimately host fitness. Five of the ten most abundant OTUs in the entire dataset belonged to a single genus, 
Rhizobiales. Rhizobiales are abundant in the guts of many fish species (Sullam et al. 2012), and decrease as diets become more carnivorous in fish (Sullam et al. 2012) as well as in ants (Russell et al. 2009). Rhizobiales can convert $\mathrm{N}_{2}$ to absorbable nutrients, and Russell et al. (2009) suggests hosts could evolve a reliance on these $\mathrm{N}$ fixers to supplement a poor quality diet. We found higher Rhizobiales abundance in guts of fish in LP environments compared to those in HP, and the highest abundances in fish translocated 30-60 years ago (old introduction). These populations tended to have diets particularly low in invertebrates (see Fig. S1) but high in detritus (data not shown), which would make them N-limited. In the guppy system, density-dependent factors are thought to underlie predictable evolution of the LP phenotype (Bassar et al. 2010). Adapting to the LP environment involves adapting to higher fish densities and lower per capita resources, which could also potentially result in strong selection for highly efficient gut microbiota. In contrast, HP populations can, and do, select for N-rich invertebrates (Zandonà et al. 2011). Perhaps in LP guts, the abundance of labile C in the form of detritus can support the energy-intensive process of N-fixation from Rhizobiales (Stoll et al. 2007), and satisfy nutrient demand.

It is intriguing that this strategy was more highly adopted in introduced populations, compared to either their sources (HP) or the native (LP) sites that we expected them to converge with (Fig. 5C). The fact that introduced populations had more sources of microbiome transmission (vertical transmission from source population, and horizontal transmission from the new LP environment) may have driven their significantly higher microbiome richness (Fig. 5A). Perhaps this greater gut diversity, representing higher 'additive variation' of the hologenome (Zilber-Rosenberg \& Rosenberg 2008), was needed to select for nutritionally-beneficial taxa, allowing older translocated populations to take better advantage of this strategy than native fish. This possible mechanism for microbiome-facilitated nutrient acquisition - taken in combination with the observed consistent divergence of microbiome away from HP source populations - points to a nutrition-related role for the microbiome in guppy evolution, even in the absence of a proximal link between gut content and microbiome. Future studies could confirm the causality of this link by manipulating microbiome and documenting changes in host fitness (see Rudman et al. (2019) for work in model organisms). Our study shows that these studies will be much more relevant to wild populations if they consider trait plasticity, realistic dietary patterns, and environmental properties all of which may co-vary with ecotype divergence.

\section{Conclusion}

We found that gut microbiome tracks host evolution in multiple independent translocations in the wild. By studying multiple stages of this co-evolution, we revealed that the gut microbiome rapidly diverges from that of source populations - with shifts in gut morphology and environment changes - and inconsistently converges with LP native populations. We argue that lack of parallel convergence does not preclude a role for the microbiome in guppy evolution; in fact, microbiomes may provide a consistent nutritional advantage to guppies introduced to low-resource environments, provided there is the sufficient richness to filter from. In contrast to much of our knowledge of host-microbiome evolution based on macro-evolutionary patterns, our study offers an unprecedented view of host-microbiome co-evolution in real-time in wild populations.

\section{Acknowledgements}

We thank Karen Sullam for helpful discussions related to project design and John Endler, Caryl Haskins, and David Reznick for initiating the original guppy introduction experiments that made this work possible. We also thank Kevin Dougherty and Lukas Bell-Dereske for assistance with laboratory and bioinformatic analyses and Nick Haddad, David Reznick, Joe Travis, and the Evans Lab for comments on earlier versions of this manuscript. We further thank David Reznick and his co-PIs from National Science Foundation Frontiers in Integrative Biological Research grant EF-0623632 for their support and intellectual contribution to our work. Funding was provided by Michigan State University and an NSF FSML grant (1722621) to SEE and SWF, Prociência UERJ and CNPq grant (308261/2017-8) to EZ and CAPES scholarship to JRA. Animal care and experimental procedures were approved by Colorado State University's Institutional Animal Care 
and Use Committee (protocol no.12-3818A) and by the Fisheries division of Trinidad's Ministry of Food Production, Land and Marine Affairs. This is Kellogg Biological Station contribution number 2173.

\section{References}

Abe, T., Bignell, D., Higashi, M., Higashi, T. \& Abe, Y. (2000).Termites: Evolution, Sociality, Symbioses, Ecology . Termit. Evol. Soc. Symbioses, Ecol.

Anderson, M. \& Walsh, D. (2013). PERMANOVA, ANOSIM, and the Mantel test in the face of heterogeneous dispersions: What null hypothesis are you testing? Ecol. Monogr. , 83, 557-574.

Bassar, R.D., Marshall, M.C., López-Sepulcre, A., Zandonà, E., Auer, S.K., Travis, J., et al. (2010). Local adaptation in Trinidadian guppies alters ecosystem processes. Proc. Natl. Acad. Sci. U. S. A., 107, 36163621.

Bates, D., Mächler, M., Bolker, B.M. \& Walker, S.C. (2015). Fitting linear mixed-effects models using lme4. J. Stat. Softw.

Beemelmanns, A., Poirier, M., Bayer, T., Kuenzel, S. \& Roth, O. (2019). Microbial embryonal colonization during pipefish male pregnancy.Sci. Rep., 9, 3.

Benson, A.K., Kelly, S. a, Legge, R., Ma, F., Low, S.J., Kim, J., et al. (2010). Individuality in gut microbiota composition is a complex polygenic trait shaped by multiple environmental and host genetic factors. Proc. Natl. Acad. Sci. U. S. A. , 107, 18933-8.

Bolnick, D.I., Snowberg, L.K., Hirsch, P.E., Lauber, C.L., Knight, R., Caporaso, J.G., et al. (2014a). Individuals' diet diversity influences gut microbial diversity in two freshwater fish (threespine stickleback and Eurasian perch). Ecol. Lett. , 17, 979-87.

Bolnick, D.I., Snowberg, L.K., Hirsch, P.E., Lauber, C.L., Org, E., Parks, B., et al. (2014b). Individual diet has sex-dependent effects on vertebrate gut microbiota. Nat. Commun. , 5, 4500.

Bordenstein, S.R. \& Theis, K.R. (2015). Host biology in light of the microbiome: Ten principles of holobionts and hologenomes. PLoS Biol., 13.

Colston, T.J. \& Jackson, C.R. (2016). Microbiome evolution along divergent branches of the vertebrate tree of life: what is known and unknown. Mol. Ecol. , 25, 3776-3800.

Endler, J.A. (1980). Natural selection on color patterns in Poecilia reticulata. Evolution , 34, 76.

Fierer, N., Morse, J.L., Berthrong, S.T., Bernhardt, E.S. \& Jackson, R.B. (2007). Environmental controls on the landscape-scale biogeography of stream bacterial communities. Ecology , 88, 2162-2173.

Fitzpatrick, S.W., Gerberich, J.C., Kronenberger, J.A., Angeloni, L.M. \& Funk, W.C. (2015). Locally adapted traits maintained in the face of high gene flow. Ecol. Lett. , 18, 37-47.

Forberg, T., Sjulstad, E.B., Bakke, I., Olsen, Y., Hagiwara, A., Sakakura, Y., et al. (2016). Correlation between microbiota and growth in Mangrove Killifish (Kryptolebias marmoratus) and Atlantic cod (Gadus morhua). Sci. Rep., 6, 21192.

Gee, H., Howlett, R. \& Campbell, P. (2009). 15 Evolutionary Gems.Nature .

Gordon, S.P., Reznick, D., Arendt, J.D., Roughton, A., Hernandez, M.N.O., Bentzen, P., et al. (2015). Selection analysis on the rapid evolution of a secondary sexual trait. Proc. R. Soc. B Biol. Sci. , 282, 20151244.

Gould, A.L., Zhang, V., Lamberti, L., Jones, E.W., Obadia, B., Korasidis, N., et al. (2018). Microbiome interactions shape host fitness. Proc. Natl. Acad. Sci., 115, 201809349.

Grether, G.F., Millie, D.F., Bryant, M.J., Reznick, D.N. \& Mayea, W. (2001). Rain forest canopy cover, resource availability, and life history evolution in guppies. Ecology, 82, 1546-1559. 
Härer, A., Torres-Dowdall, J., Rometsch, S.J., Yohannes, E., Machado-Schiaffino, G. \& Meyer, A. (2020). Parallel and non-parallel changes of the gut microbiota during trophic diversification in repeated young adaptive radiations of sympatric cichlid fish. Microbiome, 8 .

Jacobsen, D., Schultz, R. \& Encalada, A. (1997). Structure and diversity of stream invertebrate assemblages: The influence of temperature with altitude and latitude. Freshw. Biol. , 38, 247-261.

Kapoor, B.G., Smit, H. \& Verighina, I.A. (1976). The Alimentary Canal and Digestion in Teleosts . Adv. Mar. Biol., Advances in marine biology. Advances in marine biology.

Karasov, W.H. \& Douglas, A.E. (2013). Comparative digestive physiology.Compr. Physiol. , 3, 741-783.

Ke, Z., Xie, P. \& Guo, L. (2008). Phenotypic plasticity in gut length in the planktivorous filter-feeding silver carp (Hypophthalmichthys molitrix). Scientific WorldJournal. , 8, 169-175.

Kohl, K.D., Weiss, R.B., Cox, J., Dale, C. \& Dearing, M.D. (2014). Gut microbes of mammalian herbivores facilitate intake of plant toxins.Ecol. Lett. , 17, 1238-1246.

Kozich, J.J., Westcott, S.L., Baxter, N.T., Highlander, S.K. \& Schloss, P.D. (2013). Development of a dualindex sequencing strategy and curation pipeline for analyzing amplicon sequence data on the miseq illumina sequencing platform. Appl. Environ. Microbiol. , 79, 5112-5120.

Lathrop, S.K., Bloom, S.M., Rao, S.M., Nutsch, K., Lio, C.W., Santacruz, N., et al. (2011). Peripheral education of the immune system by colonic commensal microbiota. Nature , 478, 250-254.

Ley, R.E., Hamady, M., Lozupone, C., Turnbaugh, P.J., Ramey, R.R., Bircher, J.S., et al. (2008). Evolution of mammals and their gut microbes. Science, 320, 1647-1651.

Li, M., Wang, B., Zhang, M., Rantalainen, M., Wang, S., Zhou, H.,et al. (2008). Symbiotic gut microbes modulate human metabolic phenotypes. Proc. Natl. Acad. Sci. , 105, 2117-2122.

Louca, S., Jacques, S.M.S., Pires, A.P.F., Leal, J.S., Srivastava, D.S., Parfrey, L.W., et al. (2017). High taxonomic variability despite stable functional structure across microbial communities. Nat. Ecol. Evol., 1.

Magurran, A.E. (2005). Evolutionary Ecology: The Trinidadian Guppy . Evolutionary Ecology: The Trinidadian Guppy. OUP Oxford.

Martin, M. (2011). Cutadapt removes adapter sequences from high-throughput sequencing reads. EMBnet.journal , 17, 10.

McMurdie, P.J. \& Holmes, S. (2013). Phyloseq: An R Package for Reproducible Interactive Analysis and Graphics of Microbiome Census Data. PLoS One, 8.

Milani, C., Mancabelli, L., Lugli, G.A., Duranti, S., Turroni, F., Ferrario, C., et al. (2015). Exploring vertical transmission of Bifidobacteria from mother to child. Appl. Environ. Microbiol., 81, 7078-7087.

Mirarab, S., Nguyen, N., Guo, S., Wang, L.-S., Kim, J. \& Warnow, T. (2015). PASTA: Ultra-Large Multiple Sequence Alignment for Nucleotide and Amino-Acid Sequences. J. Comput. Biol. , 22, 377-386.

Muegge, B.D., Kuczynski, J., Knights, D., Clemente, J.C., González, A., Fontana, L., et al. (2011). Diet drives convergence in gut microbiome functions across mammalian phylogeny and within humans.Science , $332,970-974$.

Mugnai, R., Nessimian, J.L. \& Baptista, D.F. (2010). Manual de identificação de macroinvertebrados aquáticos do Estado do Rio de Janeiro: para atividades técnicas, de ensino e treinamento em programas de avaliação da qualidade ecológica dos ecossistemas lóticos. Technical Books Editora.

Nayak, S.K. (2010). Role of gastrointestinal microbiota in fish.

Aquac. Res. 
Oksanen, J., Blanchet, F.G., Kindt, R., Legendre, P., Minchin, P.R., O'Hara, R.B., et al. (2017). vegan: Community Ecology Package.R Packag. ver. 2.4-3 .

Pielou, E.C. (1975). Ecological Diversity . Wiley, New York, NY, USA.

R Core Team. (2017). R: A Language and Environment for Statistical Computing. R Found. Stat. Comput. Vienna, Austria .

Rawls, J.F., Mahowald, M.A., Ley, R.E. \& Gordon, J.I. (2006). Reciprocal Gut Microbiota Transplants from Zebrafish and Mice to Germ-free Recipients Reveal Host Habitat Selection. Cell , 127, 423-433.

Reznick, D.A., Bryga, H. \& Endler, J.A. (1990). Experimentally induced life-history evolution in a natural population. Nature, 346, 357-359.

Reznick, D.N., Bassar, R.D., Handelsman, C.A., Ghalambor, C.K., Arendt, J., Coulson, T., et al. (2019). Eco-evolutionary feedbacks predict the time course of rapid life-history evolution. Am. Nat., 194, 671-692.

Reznick, D.N. \& Bryga, H. (1987). Life-History Evolution in Guppies (Poecilia reticulata): 1. Phenotypic and Genetic Changes in an Introduction Experiment. Evolution. , 41, 1370.

Reznick, D.N., Rodd, F.H. \& Cardenas, M. (1996). Life-history evolution in guppies (Poecilia reticulata: Poeciliidae). IV. Parallelism in life-history phenotypes. Am. Nat.

Reznick, D.N., Shaw, F.H., Rodd, F.H. \& Shaw, R.G. (1997). Evaluation of the rate of evolution in natural populations of guppies (Poecilia reticulata). Science., 275, 1934-1937.

Roeselers, G., Mittge, E.K., Stephens, W.Z., Parichy, D.M., Cavanaugh, C.M., Guillemin, K., et al. (2011). Evidence for a core gut microbiota in the zebrafish. ISME J. , 5, 1595-1608.

Russell, J.A., Moreau, C.S., Goldman-Huertas, B., Fujiwara, M., Lohman, D.J. \& Pierce, N.E. (2009). Bacterial gut symbionts are tightly linked with the evolution of herbivory in ants. Proc. Natl. Acad. Sci. U. S. A. , 106, 21236-21241.

Schneider, C.A., Rasband, W.S. \& Eliceiri, K.W. (2012). NIH Image to ImageJ: 25 years of image analysis. Nat. Methods , 9, 671-5.

Sevellec, M., Derome, N. \& Bernatchez, L. (2018). Holobionts and ecological speciation: the intestinal microbiota of lake whitefish species pairs. Microbiome , 6, 47.

Sibly, R.M., Townsend, C.R. \& Calow, P. (1981). Strategies of digestion and defecation. In: Physiological ecology: an evolutionary approach to resource use. Sinauer Associates, pp. 109-139.

Smith, C.C.R., Snowberg, L.K., Gregory Caporaso, J., Knight, R. \& Bolnick, D.I. (2015). Dietary input of microbes and host genetic variation shape among-population differences in stickleback gut microbiota. ISME J. , 9, 2515-2526.

Spor, A., Koren, O. \& Ley, R. (2011). Unravelling the effects of the environment and host genotype on the gut microbiome. Nat. Rev. Microbiol. , 9, 279-90.

Stoll, S., Gadau, J., Gross, R. \& Feldhaar, H. (2007). Bacterial microbiota associated with ants of the genus Tetraponera. Biol. J. Linn. Soc., 90, 399-412.

Sullam, K.E., Essinger, S.D., Lozupone, C.A., O'Connor, M.P., Rosen, G.L., Knight, R., et al. (2012). Environmental and ecological factors that shape the gut bacterial communities of fish: A meta-analysis. Mol. Ecol. , 21, 3363-78.

Sullam, K.E., Rubin, B.E.E.R.R., Dalton, C.M., Kilham, S.S., Flecker, A.S. \& Russell, J.A. (2015). Divergence across diet, time and populations rules out parallel evolution in the gut microbiomes of Trinidadian guppies. ISME J. , 9, 1508-1522. 
Travis, J., Reznick, D. \& Bassar, R.D. (2014). Do eco-evo feedbacks help us understand nature? Answers from studies of the Trinidadian guppy. Adv. Ecol. Res. , 50, 1-40.

Turnbaugh, P.J. \& Gordon, J.I. (2009). The core gut microbiome, energy balance and obesity. In: Journal of Physiology . pp. 4153-4158.

Vuong, H.E., Yano, J.M., Fung, T.C. \& Hsiao, E.Y. (2017). The Microbiome and Host Behavior. Annu. Rev. Neurosci. , 40, 21-49.

Yilmaz, P., Parfrey, L.W., Yarza, P., Gerken, J., Pruesse, E., Quast, C., et al. (2014). The SILVA and "all-species Living Tree Project (LTP)" taxonomic frameworks. Nucleic Acids Res. , 42.

Zandonà, E., Auer, S.K., Kilham, S.S., Howard, J.L., López-Sepulcre, A., O’Connor, M.P., et al. (2011). Diet quality and prey selectivity correlate with life histories and predation regime in Trinidadian guppies. Funct. Ecol. , 25, 964-973.

Zandonà, E., Auer, S.K., Kilham, S.S. \& Reznick, D.N. (2015). Contrasting population and diet influences on gut length of an omnivorous tropical fish, the trinidadian guppy (poecilia reticulata).PLoS One , 10, 1-18.

Zandonà, E., Dalton, C.M., El-Sabaawi, R.W., Howard, J.L., Marshall, M.C., Kilham, S.S., et al. (2017). Population variation in the trophic niche of the Trinidadian guppy from different predation regimes.Sci. Rep. , 7, 5770 .

Zhu, L., Wu, Q., Dai, J., Zhang, S. \& Wei, F. (2011). Evidence of cellulose metabolism by the giant panda gut microbiome. Proc. Natl. Acad. Sci. , 108, 17714-17719.

Zilber-Rosenberg, I. \& Rosenberg, E. (2008). Role of microorganisms in the evolution of animals and plants: The hologenome theory of evolution.

FEMS Microbiol. Rev.

Tables

Table 1. Results of PerMANOVA models including all data.

\begin{tabular}{llllll}
\hline & Factor & SS & F & $\% \mathrm{R}^{2}$ & p-value \\
\hline Unweighted unifrac (presence absence) & Gut position & 3.8 & 18.3 & 3.2 & $<0.001$ \\
& Site category & 4.7 & 22.6 & 6.0 & $<0.001$ \\
& Site & 12.1 & 5.8 & 10.2 & $<0.001$ \\
Weighted unifrac (includes abundance) & Gut position & 0.22 & 8.2 & 1.3 & $<0.001$ \\
& Site category & 1.5 & 19.1 & 9.4 & $<0.001$ \\
& Site & 1.9 & 9.4 & 12.3 & $<0.001$ \\
\hline
\end{tabular}

\section{Figures}

Figure 1. Map illustrating sampling scheme for our study on the island of Trinidad, and information for each site. Six translocation scenarios are indicated by dashed grey arrows with the names of rivers and year of translocation. Black squares represent high-predation source sites and yellow stars represent translocation sites. Native LP sites (green circles from west to east) are: Tumbasson, Naranjo, and Campo. Table shows sites and sample descriptions. Footnotes indicate: ${ }^{+}$source of Turure introductions was Guanapo HP source, but is not located in this drainage. ${ }^{\alpha}$ for microbiome characterization, each gut was divided into anterior and posterior sections. 


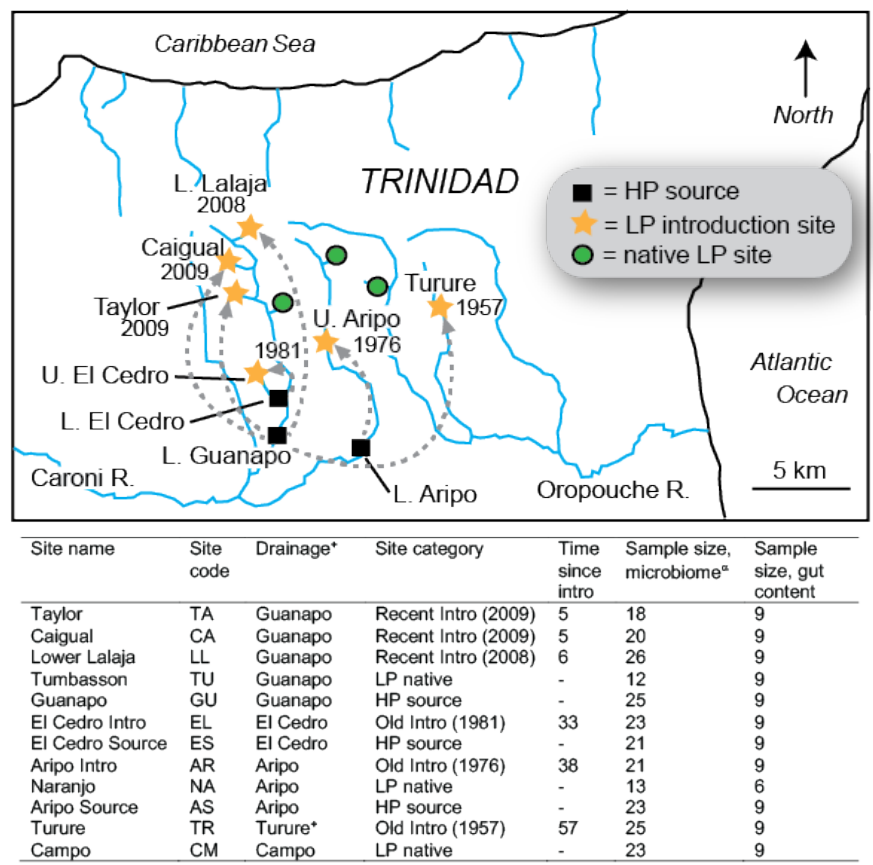

Figure 2. Stream properties, fish traits, and diet by site category. Box plots show median (solid line), upper and lower quartiles (grey box), 95\% confidence intervals (error bars), and outliers (open circles) P-values test the null hypothesis of no difference in site category (see Data analysis for univariate model details). Fig. S1 expands data to show all sites within categories.

\section{Hosted file}


image2.emf available at https://authorea.com/users/378777/articles/495181-gut-microbiomediverges-quickly-with-rapid-host-evolution-in-trinidadian-guppies

Figure 3 . Distance-based redundancy analysis based on the composition of bacterial guppy gut microbiomes (unweighted unifrac) in high predation streams, and in low predation streams, where HP fish (HP source, red) have been introduced either 30-60 years ago (old Intro, green) or 5-6 years ago (recent Intro, blue), and evolved to resemble native LP fish (black). (A) shows all site categories and (B) excludes HP source sites, showing sites in LP environments (LP native, old intro, and recent intro). Arrows show the predictor variables that explain changes in composition based on dbRDA, and length corresponds to dbRDA $\mathrm{R}^{2}$. Inset shows the partial $\mathrm{R}^{2}$ variance partitioned for diet, gut length, and water temperature (grayscale) on unconstrained data. Table S2 shows dbRDA statistics and Table 1 and S3 show PerMANOVA results.

\section{Hosted file}

image3.emf available at https://authorea.com/users/378777/articles/495181-gut-microbiomediverges-quickly-with-rapid-host-evolution-in-trinidadian-guppies

Figure 4. Dissimilarity (unweighted unifrac distance) of the gut microbiome of each population compared to the gut microbiome of its HP source population (shown by a red line at 0). Higher values are more dissimilar to HP source. Inset of (A) shows finer scale axes. Note that TR is sourced from the Guanapo HP site but because they are both in Oropouche drainage, serves as a comparison to Campo

A. Guanapo
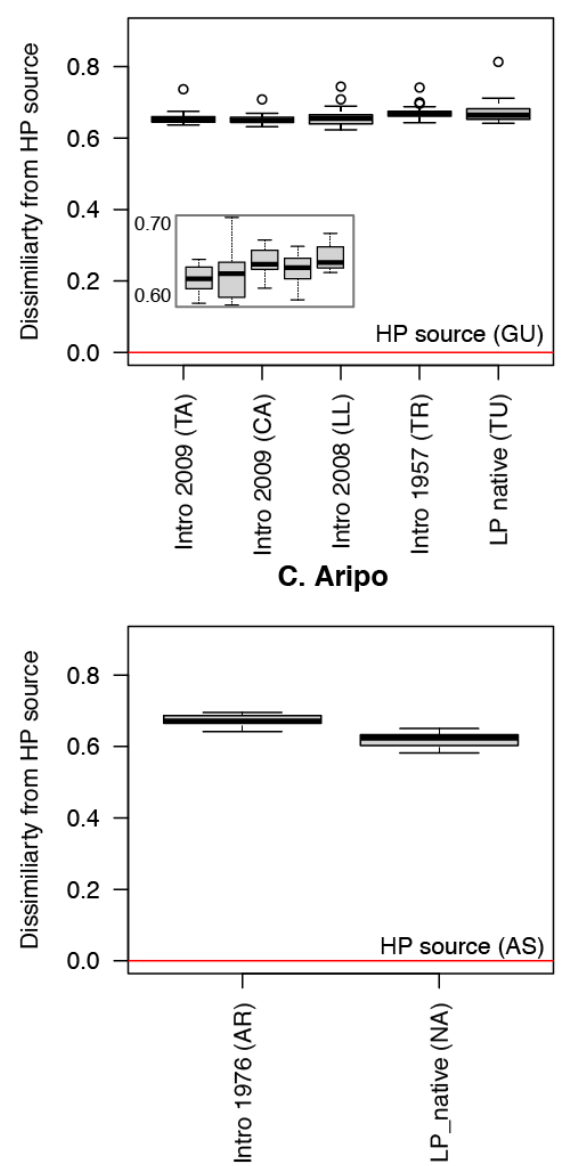

B. El Cedro
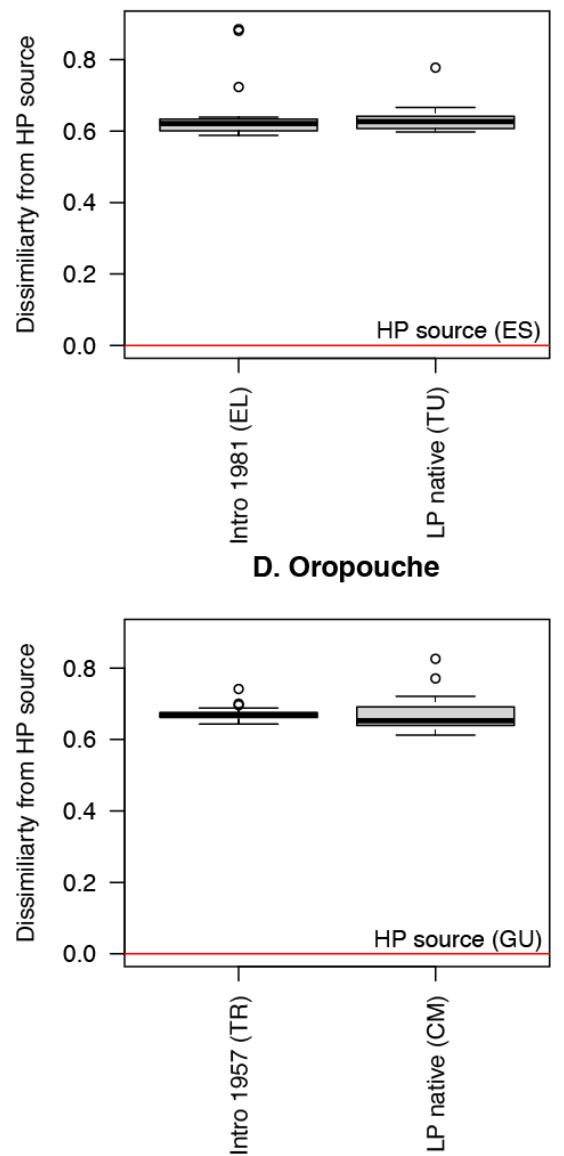

Figure 5. Gut microbiome richness (A), mean relative abundance of all Rhizobiales (B), and relative 
abundance of five Rhizobiales species (categorized by genus, if known) that were part of the ten most abundant OTUs across all treatments (C). Other community diversity metrics are shown in Fig. S4 and S5, most abundant OTUs listed in Table S4, and Rhizobiales by site is shown in Fig. S9. 


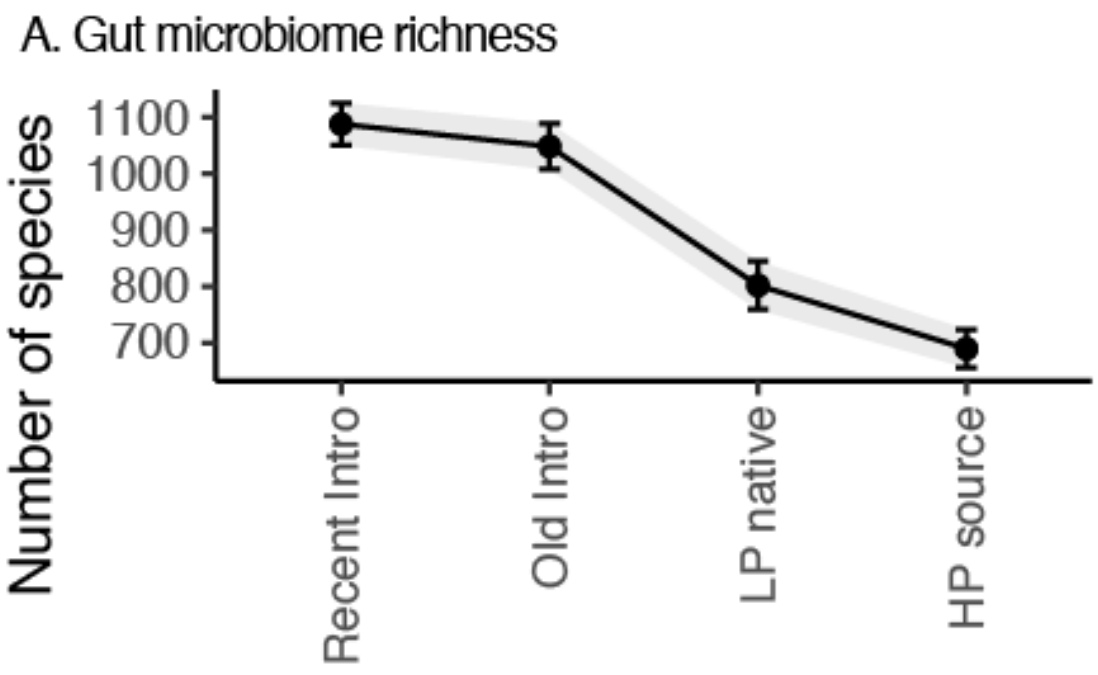

B. Abundance of Order Rhizobiales

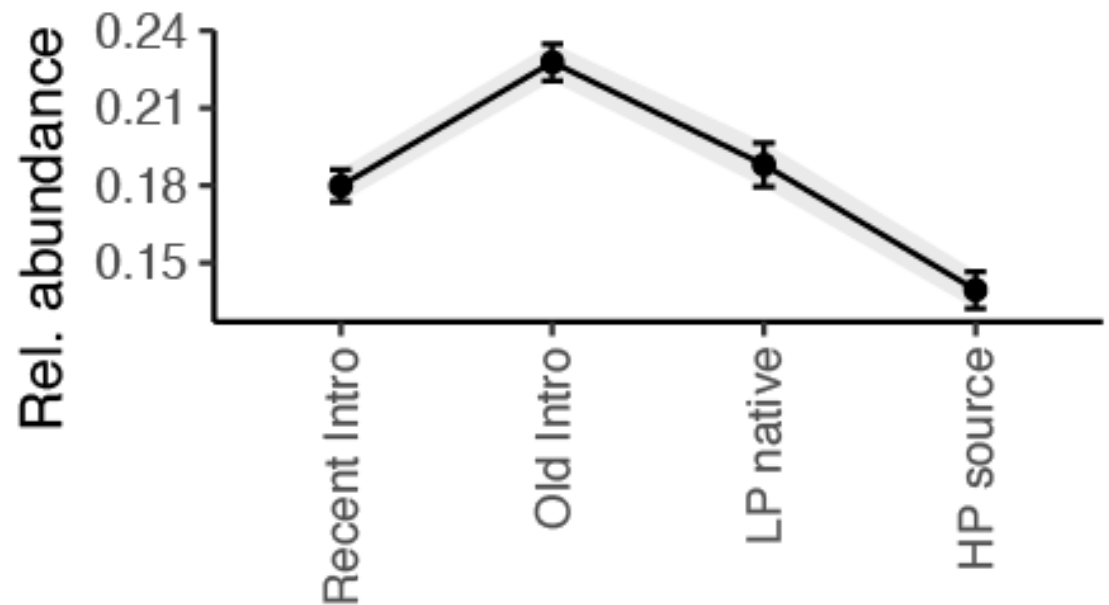

$-\quad$ Unknown (OTU4)

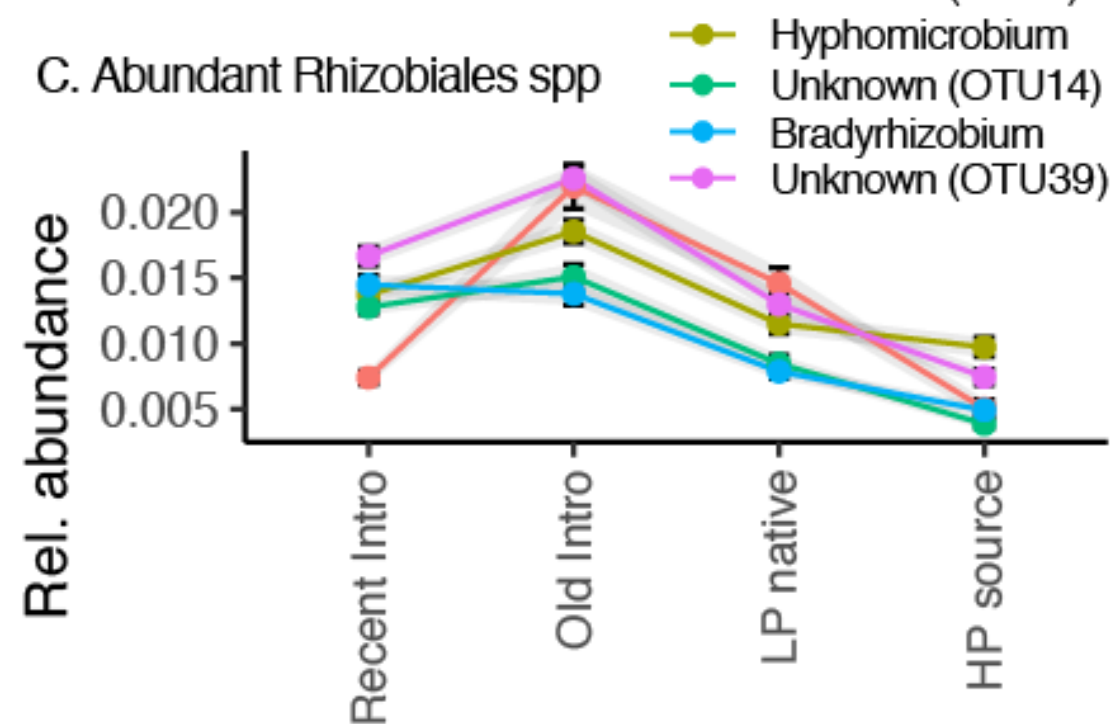

